\title{
ANALYSE DE LA VARIABILITÉ DE LA CROISSANCE D'UNE POPULATION DE TRUITE COMMUNE (SALMO TRUTTA L.) DANS UN TORRENT PYRÉNÉEN.
}

\author{
T. LAGARRIGUE (1), P. BARAN (2), J.M. LASCAUX (3) ET A. BELAUD (1)
}

(1) Ecole Nationale Supérieure Agronomique de Toulouse, Laboratoire Environnement Aquatique et Aquaculture, Avenue de l'Agrobiopole, BP 107 Auzeville-Tolosane, 31326 Castanet-Tolosan Cedex, France.

Adresse à utiliser pour la correspondance: Cabinet d'études IMAGO, 26 rue Dels Pibouls, Labastide Falgarde, 31120 Lacroix Falgarde, France.

E-mail : ecogea@wanadoo.fr

(2) Conseil Supérieur de la Pêche, DR n`5, 20 rue Charrue, 21000 Dijon, France.

(3) Cabinet d'Etudes et Conseil en Gestion de l'Environnement Aquatique, 26 rue Dels Pibouls, Labastide Falgarde, 31120 Lacroix Falgarde, France.

Reçu le 08 août 2000

Accepté le 18 octobre 2000

Received 08 August, 2000

Accepted 18 October, 2000

\section{RÉSUMÉ}

La population de truite commune de la Neste d'Oueil, sous - affluent pyrénéen de la Garonne, a été suivie entre juillet 1996 et mars 1999. Durant 2 ans et 9 mois, 9 échantillonnages ont eu lieu en février-mars, juillet et septembre sur 2 secteurs de rivière, afin de suivre l'évolution saisonnière de la croissance, de la densité totale et de la biomasse totale en truites communes.

Parallèlement à l'étude de la population, 652 truites communes d'âge compris entre $1+$ et $6+$ ans, pour des tailles comprises entre 100 et $290 \mathrm{~mm}$, ont été marquées en mars 1998 par implants visibles numérotés afin de suivre l'évolution de la croissance individuelle. Les taux de recapture entre campagne ont varié de 19.5 à $74 \%$, suivant la saison considérée.

La croissance des truites communes présente une forte variabilité saisonnière, validée par le marquage. Elle est quasiment nulle, avec des pertes de poids pour les individus d'âge $>1+$, en automne et en hiver, en raison de températures rigoureuses et d'un probable effet de la reproduction. Elle se réalise quasi exclusivement au printemps, avant d'atteindre l'optimum thermique de croissance. Elle est très faible en période estivale, malgré les conditions thermiques optimales.

En été, le ralentissement de la croissance semble résulter de l'action conjuguée de la maturation gonadique, de la saturation de la capacité d'accueil du milieu physique et de la compétition intra-spécifique maximale à cette période du fait i) de l'arrivée probable dans le cours principal d'individus 1+ et 2+ dévalants des affluents et ii) de la diminution de la "quantité et de la qualité" de l'habitat physique à l'étiage régulant l'accessibilité à la ressource trophique potentiellement disponible abondante en densité mais constituée de très petites proies, peu rentables énergétiquement.

Le rôle de la croissance dans la dynamique de la population de truite commune de la Neste d'Oueil est discuté.

Mots-clés : croissance, truite commune, Salmo trutta, marquage individuel, variabilité saisonnière, Pyrénées, France. 


\title{
GROWTH VARIABILITY ANALYSIS OF A BROWN TROUT (SALMO TRUTTA L.) POPULATION IN A PYRENEAN MOUNTAIN STREAM.
}

\begin{abstract}
The brown trout population of the Neste d'Oueil, a Pyrenean tributary of the Garonne river, was studied between July 1996 and March 1999. During the period of two years and nine months, nine sampling experiments were carried out in February-March, July and September on two sectors of the river. Thus, we could follow the seasonal variation of growth, total density and total biomass of brown trout.
\end{abstract}

Moreover, 652 brown trouts of age ranging between $1+$ and $6+$ years, for sizes ranging between 100 and $290 \mathrm{~mm}$, were marked individually by visible implants in March 1998. The recapture rates observed varied from 19.5 to $74 \%$, following the season considered.

Growth rates showed a strong seasonal variability, validated by individual tagging experiments. Because of reproduction and rigorous temperatures experienced by trout during autumn and winter, growth rates observed were almost null and individuals $>1+$ lost weight during this period. In spite of theoretically non-optimal thermal conditions, growth occurred almost exclusively in spring. Poor growth rates observed during summer are not due to thermal factor which was theoretically optimal for growth in this season and could be attributed to the sexual maturation, to the saturation of the carrying capacity of physical habitat and to the strong intraspecific competition caused by i) likely arrivals of $1+$ and 2+ individuals emigrating from tributaries and ii) reduction in "quantity and quality" of physical habitat at low-flow limiting accessibility and availability of energetically profitable prey.

The role of growth in the dynamics of the brown trout population of the Neste d'Oueil River is discussed.

Key-words : growth, brown trout, Salmo trutta, individual marking techniques, seasonal variability, Pyrenees, France.

\section{INTRODUCTION}

Dans un cadre de connaissances très ponctuelles et incomplètes de la croissance des populations de truite commune (Salmo trutta L.) pyrénéennes (ANGELIER, 1976 ; CHAUVET, 1983), une première étape a consisté à caractériser les paramètres mésologiques et l'influence de certaines activités humaines sur la croissance à une échelle globale. Ainsi, en considérant 215 sites répartis sur 84 cours d'eau de la chaîne pyrénéenne, LAGARRIGUE et al. (2001) ont montré que la taille à 3 ans des truites communes était 1) extrêmement variable selon les sites (de 128 à $314 \mathrm{~mm}$ ), du fait de la très grande diversité écologique rencontrée dans les cours d'eau de cette région, 2) fortement structurée par le gradient altitudinal, ce dernier étant intégrateur d'un gradient thermique et physico-chimique, 3) pénalisée dans les secteurs soumis à des débits réservés.

La deuxième étape consistait donc à s'intéresser à une population de truite commune sur un cours d'eau 1) pour analyser la variabilité de la croissance au niveau de la population au cours d'une année et 2) pour essayer de déterminer le rôle de la croissance dans la dynamique d'une population de truite commune dans une rivière pyrénéenne. Cette analyse a été effectuée en prenant en compte un certain nombre de 
paramètres mésologiques (température) et biotiques (ressources trophiques, compétition intra-spécifique) sur un cours d'eau pyrénéen qui a fait l'objet d'un suivi depuis plusieurs années (GOURAUD, 1999 ; GOURAUD et al., 1999).

\section{MATÉRIEL ET MÉTHODES}

\section{Présentation du secteur d'étude}

L'étude s'est déroulée sur un sous - affluent du bassin Pyrénéen de la Garonne, la Neste d'Oueil (Figure 1). Cette rivière est issue de la réunion à $1.450 \mathrm{~m}$ d'altitude de plusieurs ruisseaux prenant leur source à environ $1850 \mathrm{~m}$ d'altitude. Elle conflue avec l'One à 765 m d'altitude, après un parcours de $9.2 \mathrm{~km}$. Sur le bassin-versant orienté NO $\mathrm{SE}$, alternent forêts et prairies. La pente moyenne de la rivière est de 11.8 \% et la largeur varie de $4 \mathrm{~m}$ à $7 \mathrm{~m}$. Le régime hydrologique est de type nivo-pluvial avec un étiage estival et deux périodes de fortes eaux durant la fonte des neiges printanière et les fortes pluies automnales. Le module est de l'ordre de 380 I/ s dans la partie amont et de 1085 I / s dans la partie aval (GOURAUD, 1999). Les eaux sont exemptes de toute pollution organique ou chimique, la pression anthropique étant très faible sur l'ensemble du bassin versant.

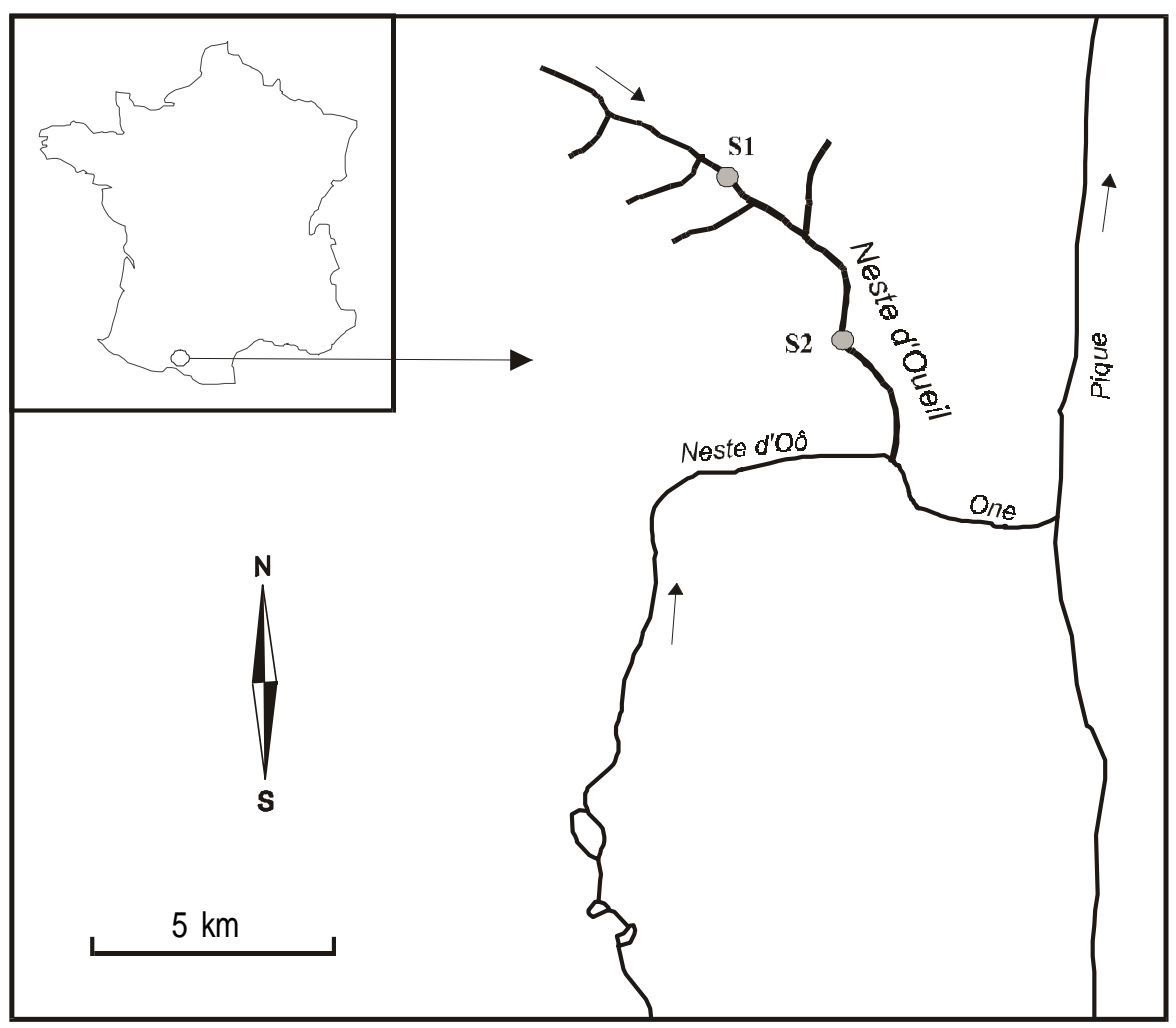

\section{Figure 1}

Situation géographique de la Neste d'Oueil dans les Pyrénées et localisation des deux stations d'étude.

\section{Figure 1}

Geographical situation of the river Neste d'Oueil in the Pyrenees and localization of the two sampling sites. 
Au cours d'une reconnaissance morphodynamique complète de la rivière, le pourcentage de représentativité des différents groupes de faciès d'écoulement, définis selon la classification de DELACOSTE et al. (1995), a été établi. A partir de cette reconnaissance morphodynamique, deux stations d'étude S1 et S2 ont été sélectionnées, afin d'être représentatives respectivement du secteur de plateau amont et du secteur montagnard aval. Leurs principales caractéristiques sont présentées dans le Tableau I.

\section{Tableau I}

Principales caractéristiques physiques et chimiques relevées en juillet 1996 aux deux stations.

\section{Table I}

Main physical characteristics and chemical description of the two sites in July 1996.

\begin{tabular}{|c|c|c|}
\hline Caractéristiques physiques et chimiques & Station 1 - amont & Station 2 - aval \\
\hline Altitude (m) & 1247 & 1060 \\
\hline Longueur $(\mathrm{m})$ & 151 & 178 \\
\hline Largeur moyenne $(\mathrm{m})$ & 3.5 & 5.5 \\
\hline Distance à la source $(\mathrm{km})$ & 4.3 & 6.4 \\
\hline Pente du secteur (\%) & 2 & 4.4 \\
\hline Principaux types de faciès d'écoulement & Radier, Plat, Escalier & $\begin{array}{c}\text { Pool, Escalier, Rapide } \\
\text { Plat }\end{array}$ \\
\hline $\mathrm{pH}$ & 7.2 & 7.4 \\
\hline Conductivité estivale ( $\mu \mathrm{S} / \mathrm{cm})$ & 170 & 152 \\
\hline Oxygène (mg / l) & 10.9 & 9.8 \\
\hline Bicarbonates $\left(\mathrm{mg} \mathrm{HCO}_{3}^{-} / \mathrm{l}\right)$ & & 101 \\
\hline Nitrates (mg NO$\left.{ }_{3}^{-} / \mathrm{l}\right)$ & & 1.8 \\
\hline Phosphates (mg PO$\left.{ }_{4}^{--} / \mathrm{l}\right)$ & & $<0.05$ \\
\hline Sulfates $\left(\mathrm{mg} \mathrm{SO}_{4}^{-} / \mathrm{I}\right)$ & & 8 \\
\hline Chlorures (mg Cl / I) & & $<1$ \\
\hline Calcium (mg Ca $\left.a^{++} / \mathrm{l}\right)$ & & 35 \\
\hline Magnésium $\left(\mathrm{mg} \mathrm{Mg}^{++} / \mathrm{l}\right)$ & & 2.2 \\
\hline Sodium (mg Na / I) & & 2 \\
\hline Potassium (mg K+ / I) & & 0.4 \\
\hline
\end{tabular}

\section{Récolte et traitement des données}

\section{Température et macrofaune benthique}

La température de l'eau a été enregistrée sans interruption au moyen de deux thermographes de type PEKLY indic 8000 à pas horaire, installés aux deux stations.

Au cours des campagnes de juillet et septembre 1998, des prélèvements exhaustifs de macrofaune benthique ont été effectués afin d'approcher la ressource trophique potentiellement disponible aux deux stations. Pour cela, 3 faciès d'écoulement représentatifs et répartis sur l'ensemble de chaque station ont été choisis et les prélèvements ont été effectués à l'aide d'un filet Surber (surface d'échantillonnage de $1 / 10^{\text {ème }}$ de $\mathrm{m}^{2}$ ), à raison de 4 prélèvements par faciès. Les densités et les biomasses des 
principales familles d'invertébrés benthiques présentes dans chaque station ont ainsi été estimées.

\section{Populations de poissons}

\section{Population totale (période de juillet 1996 à mars 1999)}

Les peuplements piscicoles des 2 stations ont été inventoriés par pêche électrique à 9 reprises pendant les 2 ans et 9 mois de suivi, en juillet et septembre 1996, en février, juillet et septembre 1997, en mars, juillet et septembre 1998, et en mars 1999. Selon la saison, la conductivité de l'eau s'échelonnait entre 120 et $200 \mu \mathrm{S} / \mathrm{cm}$. Deux passages successifs ont été effectués à effort de pêche constant (2 électrodes), conformément à la méthode de DE LURY (1951). Les poissons ont été mesurés (longueur totale, au mm près) et pesés (au g près) lors de toutes les campagnes. Des écailles ont été prélevées sur la zone standard de 50 individus par station afin de déterminer leur âge par scalimétrie (OMBREDANE et BAGLINIÈRE, 1992). Cinq à huit écailles non régénérées par individu ont été nettoyées et lues sur un écran de rétroprojecteur (grossissement $x$ 25). La taille moyenne et le poids moyen par classe d'âge ont ainsi été déterminés.

Les densités totales par station ont été estimées aux différentes campagnes (sauf en mars 1999) par la méthode de SEBER et LE CREN (1967), les calculs étant séparés pour les individus $0+$ et les individus plus âgés. Les biomasses ont ensuite été évaluées.

Pour chaque cohorte, le taux de croissance journalier en poids, $G_{p}$ (JOBLING, 1994), a été calculé à chaque saison, à l'exception de la cohorte $0+$, selon la formule :

$$
G_{P}=\left[\left(\operatorname{Ln} P_{f}-\operatorname{Ln} P_{i}\right) /\left(t_{f}-t_{i}\right)\right] * 100
$$

avec $G_{p}$ en \% d'accroissement du poids corporel par jour, $P_{f}\left(P_{i}\right)$ les poids moyens (en g) aux temps $t_{f}\left(t_{i}\right)$. Ce taux de croissance journalier nous permet de nous affranchir de la différence de nombre de jours écoulés entre campagnes.

Le coefficient de condition de Fulton (K), ainsi que le Coefficient Ecologique de Croissance (CEC ou EGC ; PREALL et RINGLER, 1989) ont été calculés par classe d'âge, à chaque saison. Le CEC exprime le ratio entre le taux de croissance en poids observé $\left(G_{\text {obs }}\right)$ et le taux de croissance en poids maximal théorique $\left(G_{\max }\right)$ prédit par l'utilisation du modèle thermique de croissance établit par ELLIOTT et al. (1995), tel que :

$$
C E C=\left[\mathrm{G}_{\mathrm{obs}} / \mathrm{G}_{\max }\right] \times 100
$$

avec $G_{\text {obs }}=G_{p}$ et $G_{\max }$ exprimés en pourcentage d'accroissement du poids corporel par jour, la température moyenne mensuelle relevée à chaque station étant utilisée pour l'application du modèle d'ELLIOTT et al. (1995).

Le CEC permet d'évaluer "le rendement écologique" de la croissance (par rapport à une référence standard établie en laboratoire) et de s'affranchir du facteur thermique afin d'identifier le rôle d'autres facteurs écologiques sur la croissance des poissons (PREALL et RINGLER, 1989).

\section{Individus marqués (période de mars 1998 à mars 1999)}

652 truites communes de taille > $100 \mathrm{~mm}$ ont été marquées individuellement le 02 / 03/ 1998 par implants visibles numérotés (Northwest Marine Technology, Inc.), insérés au moyen d'une seringue sous l'épiderme transparent de la bordure postérieure du globe oculaire (BRYAN et NEY, 1994 ; LE LOUARN et BAGLINIERE, 1997). Des écailles 
ont été prélevées sur chaque individu marqué afin de déterminer leur âge par scalimétrie. Les recaptures ont été effectuées le 10 / 07 / 1998, le 29 / 09 / 1998 puis le 03 / 03 / 1999.

Comme pour l'ensemble de la population, les taux de croissance journaliers en poids $\left(G_{p}\right)$, les coefficients de condition de Fulton $(K)$ et les Coefficients Ecologiques de Croissance $(C E C)$ ont été calculés par classes d'âge et par campagne, en considérant l'ensemble des individus marqués puis recapturés à chaque campagne.

\section{Analyse statistique}

Les tailles moyennes et les poids moyens par âge, les taux de croissance journaliers en poids, les coefficients de condition, les $C E C$ et les densités et biomasses d'invertébrés benthiques ne suivent pas une distribution normale (test de Lilliefors, $p<$ 0.05). Ils ont été comparés entre stations, campagnes ou saisons, classes d'âge ou entre individus marqués et non marqués, par analyse de variance à un facteur sur les rangs (test de Kruskal-Wallis) suivie, le cas échéant, d'un test de comparaison par paires (test $U$ de Mann-Whitney) (SOKAL et ROHLF, 1997). Tout au long de l'analyse, le seuil de significativité à été fixé à $p<0.05$.

\section{RÉSULTATS}

\section{Régime thermique}

Les profils thermiques aux deux stations restent très similaires d'une année sur l'autre (Figure 2), même si les températures moyennes mensuelles, enregistrées entre juillet 1996 et mars 1999, étaient supérieures en 1997, par rapport à celles enregistrées en 1996, 1998 et 1999. Ces moyennes mensuelles varient entre $3.7^{\circ} \mathrm{C}$ (en janvier 1998) et $14^{\circ} \mathrm{C}$ (en août 1997) à l'amont et entre $5.1^{\circ} \mathrm{C}$ (en janvier 1999) et $14.9^{\circ} \mathrm{C}$ (en août 1997) à l'aval. Les températures moyennes journalières varient entre $1.3^{\circ} \mathrm{C}$ (en janvier 1998) et $16.3^{\circ} \mathrm{C}$ (en août 1997$)$. L'écart de température moyen entre l'amont et l'aval est de $1.1^{\circ} \mathrm{C}$.
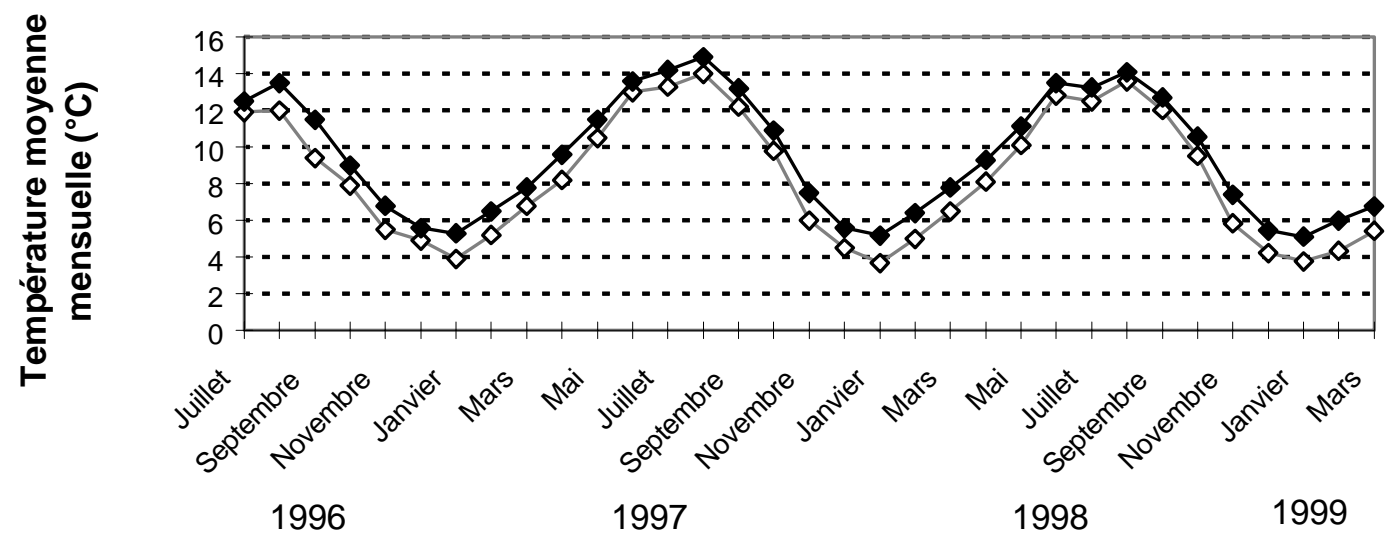

Figure 2

Evolution de la température moyenne mensuelle $\left({ }^{\circ} \mathrm{C}\right)$, relevée entre juillet 1996 et mars 1999, à la station 1 amont (losanges vides) et à la station 2 aval (losanges pleins).

\section{Figure 2}

Observed mean monthly water temperature ( $\left.{ }^{\circ} \mathrm{C}\right)$, from July 1996 to March 1999, at site 1 upstream (blank diamonds) and at site 2 downstream (solid diamonds). 


\section{Macrofaune benthique}

Les densités et les biomasses en invertébrés benthiques sont significativement plus élevées (Mann-Whitney, $p<0.001$ et $p<0.005$ respectivement) en juillet qu'en septembre. II n'y a pas de différence significative entre stations (Mann-Whitney, $p>0.05$ ), sauf pour la biomasse totale en juillet qui est plus élevée à la station 1 (Mann-Whitney, $p<0.05$ ).

Les densités estivales sont considérables (Tableau II) et il existe un facteur 6 entre les densités de juillet et de septembre, sur les deux stations. Pourtant, cette grande différence de densités ne se retrouve pas au niveau des biomasses puisque il n'y a qu'un facteur 2 entre les biomasses de juillet et de septembre. Ceci témoigne donc d'une ressource trophique très abondante en densités mais constituée d'individus de très petite taille en juillet et de proies, certes moins nombreuses en septembre, mais de plus grande taille. Ainsi, la taille des individus en juillet, et notamment celle des principales familles d'Ephéméroptères et de Plécoptères représentées, est extrêmement réduite pour la saison et ce type de cours d'eau de montagne (REYES-MARCHANT, communication personnelle).

\section{Tableau II}

Densités moyennes et biomasses moyennes d'invertébrés benthiques relevées en juillet et en septembre 1998 aux deux stations.

\section{Table II}

Mean total density and biomass of benthic macroinvertebrates in July and September 1998, at the two sites.

\begin{tabular}{|l|c|c|c|c|}
\cline { 2 - 5 } \multicolumn{1}{c|}{} & \multicolumn{2}{c|}{ Station 1 - amont } & \multicolumn{2}{c|}{ Station 2 - aval } \\
\cline { 2 - 5 } \multicolumn{1}{c|}{} & $\begin{array}{c}\text { Densité } \\
\left.\text { (ind. / } \mathbf{~}^{2}\right)\end{array}$ & $\begin{array}{c}\text { Biomasse } \\
\left(\mathbf{g} / \mathbf{~ m}^{\mathbf{2}}\right)\end{array}$ & $\begin{array}{c}\text { Densité } \\
\left.\text { (ind. / } \mathbf{~}^{\mathbf{2}}\right)\end{array}$ & $\begin{array}{c}\text { Biomasse } \\
\left(\mathbf{g} / \mathbf{~ m}^{\mathbf{2}}\right)\end{array}$ \\
\hline Juillet 1998 & 16130 & 13.95 & 12967 & 8.3 \\
\hline Septembre 1998 & 2748 & 6.13 & 2088 & 4.74 \\
\hline
\end{tabular}

Les principales familles dominant le peuplement en densité sont :

- en juillet : les Baetidae (60.7\%), Leuctridae (8.8\%), Nemouridae $(8.2 \%)$ et Simulidae $(5.1 \%)$ pour la station 1 et les Baetidae $(35.7 \%)$, Simulidae $(17.7 \%)$ et Chironomidae (3.2\%) pour la station 2.

- en septembre : les Leuctridae (30.8\%), Baetidae (16.2\%) et Elmidae $(13.9 \%)$ pour la station 1 et les Heptageniidae (35\%), Simulidae ( $9.8 \%)$ et Elmidae $(9.5$ \%) pour la station 2.

\section{Population totale : période de juillet 1996 à mars 1999}

Le peuplement piscicole de la Neste d'Oueil n'est composé que de truites communes dans le secteur étudié. Les densités totales varient entre 2021 et 11516 ind. / ha suivant les stations et les campagnes. De même pour les biomasses totales qui varient entre 94.6 et $212.5 \mathrm{~kg} / \mathrm{ha}$.

Quelle que soit l'année considérée, l'évolution saisonnière de la densité totale (Figure 3 ) est similaire à la station 1 : elle augmente progressivement entre juillet et 
septembre puis diminue entre septembre et février/mars. A la station 2, elle est plus variable : si elle diminue toujours entre septembre et février/mars, elle peut diminuer ou augmenter entre juillet et septembre.

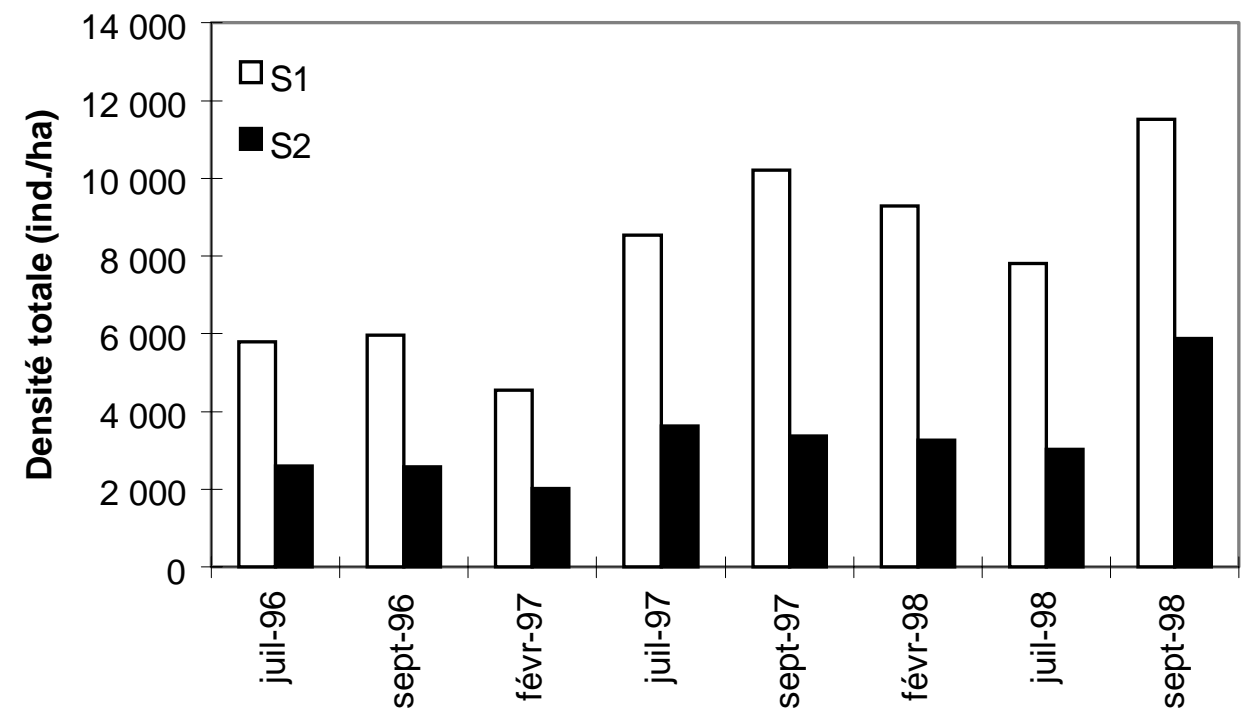

\section{Figure 3}

Evolution saisonnière de la densité totale aux deux stations, entre juillet 1996 et septembre 1998.

\section{Figure 3}

Seasonal variations of the total density at the two sites between July 1996 and September 1998.

Etant donné les difficultés de capture et de pesée des individus d'âge $0+$, notamment pour les campagnes de juillet en raison de leur petite taille, ainsi que les faibles densités d'individus d'âge $>4+$, la suite de l'analyse sera consacrée aux individus d'âge compris entre $1+$ et $4+$ ans.

\section{Taux de croissance journalier en poids}

Les taux de croissance journaliers en poids ne sont pas différents (Mann-Whitney, $p>0.05$ ) entre stations, quelle que soit la saison considérée. Par contre, pour une saison donnée, ils sont significativement différents entre classes d'âge (Kruskal-Wallis, $p<0.05$ ). Ils sont toujours plus élevés chez les individus d'âge 1+ et diminuent généralement avec l'âge des individus.

Indépendamment d'une variabilité inter-annuelle (Figure 4), il existe une évolution saisonnière très comparable d'une année à l'autre, les taux de croissance journaliers en poids étant significativement différents (Kruskal-Wallis, $p<0.001$ ) entre saisons, pour toutes les classes d'âge. Ainsi, ce taux de croissance est toujours le plus élevé entre février (ou mars) et juillet, peu élevé entre juillet et septembre et le plus faible entre septembre et février (ou mars), pour toutes les classes d'âge. Au cours de cette dernière saison, toutes les classes d'âge $>1+$ perdent du poids, ainsi que les 3+ et 4+ entre juillet et septembre 1998 à la station 2. 

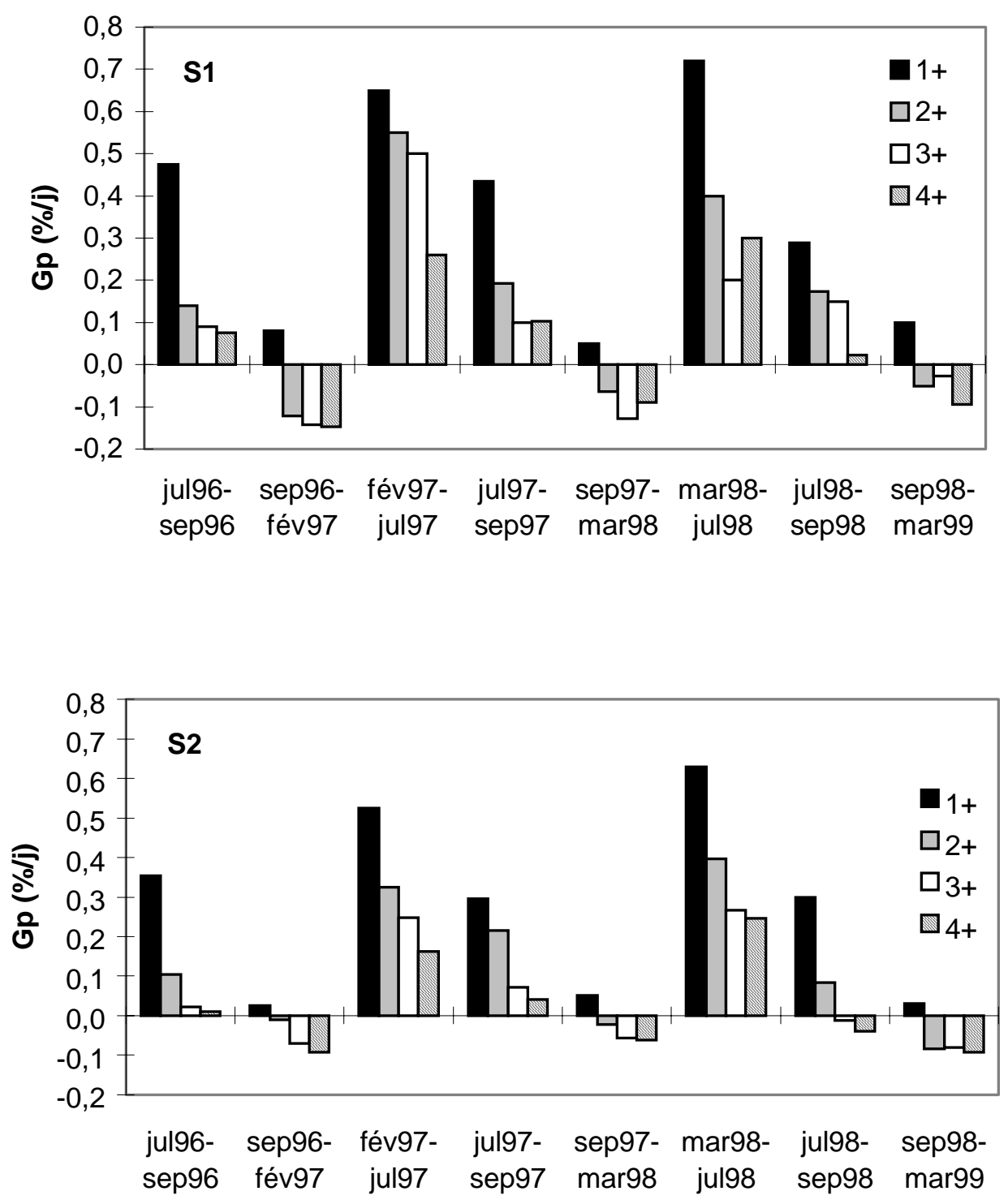

\section{Figure 4}

Evolution saisonnière des taux de croissance journaliers moyens en poids par classes d'âge (Gp), évalués pour la population totale et aux deux stations, entre juillet 1996 et mars 1999.

\section{Figure 4}

Seasonal variations of mean daily growth rates in weight by age classes (Gp), evaluated for the total population and at the two sites, between July 1996 and March 1999.

\section{Coefficients de condition}

Toutes campagnes confondues, les coefficients de condition sont significativement différents (Mann-Whitney, $\mathrm{p}<0.05$ ) entre stations. Par contre, si on les compare campagne par campagne, ils sont significativement différents (Mann-Whitney, $p<0.05$ ) 
entre stations pour les campagnes de 1996 et 1997, mais pas pour les campagnes de 1998 et de 1999 (Mann-Whitney, $p>0.05$ ). Ainsi, ils ont tendance à être plus élevés à la station 2 qu'à la station 1 en 1996 et 1997, mais pas en 1998 et 1999 (Figure 5). Pour une campagne donnée, les coefficients de condition ne sont pas significativement différents entre classes d'âge (Kruskal-Wallis, $p>0.05$ ).
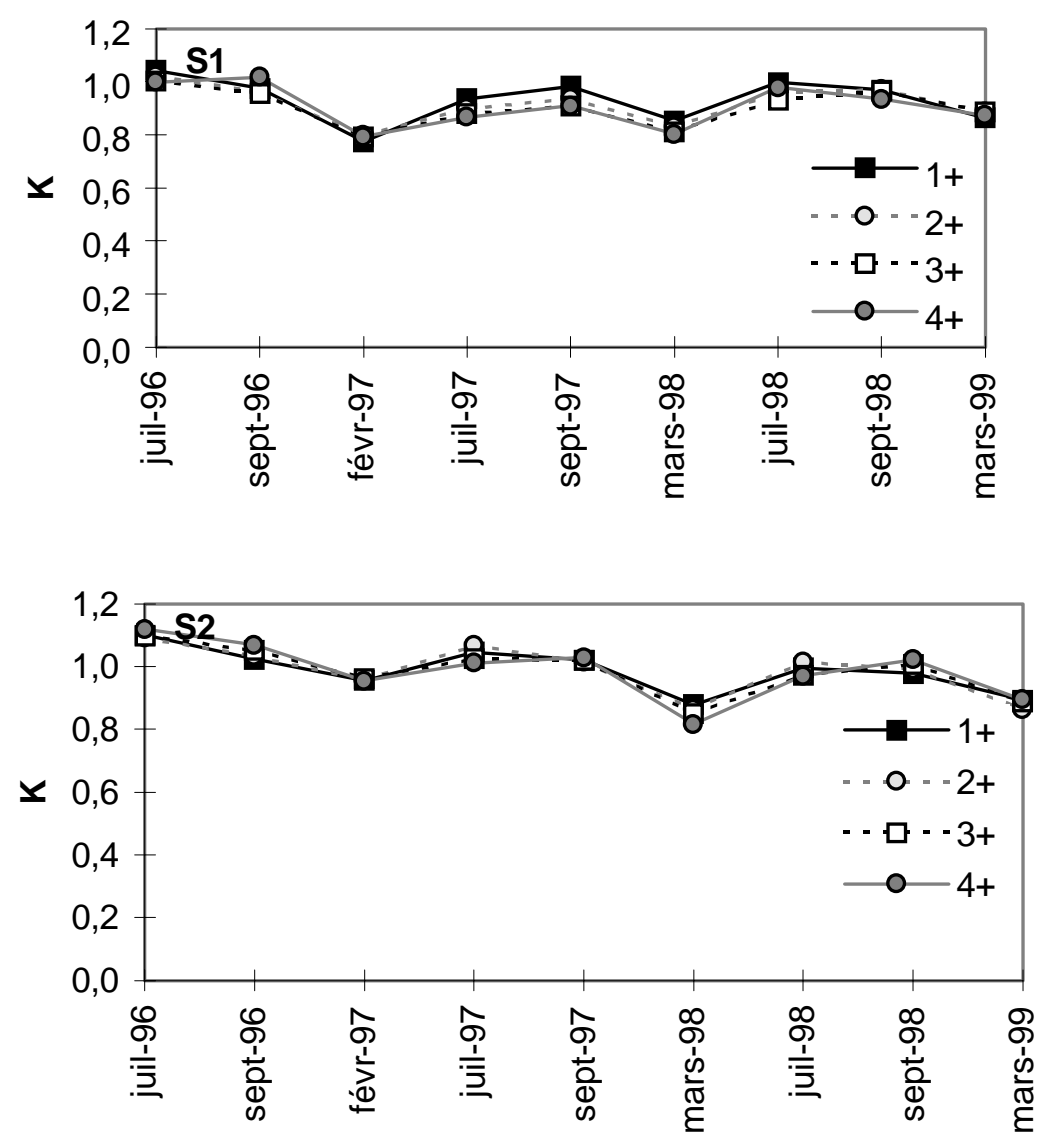

\section{Figure 5}

Evolution saisonnière des coefficients de condition moyens par classes d'âge (K), évalués pour la population totale et aux deux stations, entre juillet 1996 et mars 1999.

\section{Figure 5}

Seasonal variations of mean condition factor by age classes (K), evaluated for the total population and at the two sites, between July 1996 and March 1999.

Malgré cette différence inter-stationnelle selon les campagnes et une variabilité inter-annuelle, il existe une évolution saisonnière très comparable d'une station à l'autre et d'une année à l'autre. Homologues en juillet et en septembre, les coefficients de condition ont tendance à être plus faibles en février (ou mars), pour toutes les classes d'âge.

\section{Coefficients Ecologiques de Croissance (CEC)}

Nous avons montré que les taux de croissance journaliers en poids n'étaient pas significativement différents (Mann-Whitney, $p>0.05$ ) entre stations, quelle que soit la saison considérée. En outre, l'écart moyen de température sur une année de $1.1^{\circ} \mathrm{C}$ entre 
les deux stations, n'entraîne que des différences d'estimation du taux de croissance en poids maximal théorique prédit par le modèle d'ELLIOTT et al. (1995) de l'ordre de 8 à 10 $\%$ et de $C E C$ de l'ordre de 6 à $8 \%$, selon la classe d'âge considérée. Nous avons donc regroupé les individus des deux stations pour estimer les CEC par classe d'âge, pour l'ensemble du secteur de rivière étudié.

Les CEC sont toujours inférieurs à $100 \%$, pour toutes les saisons et pour toutes les classes d'âge, hormis pour la classe d'âge 1+ entre mars et juillet 1998 (Figure 6). Ce ne sont donc pas les valeurs de $C E C$ en elles-mêmes qui nous intéressent mais leurs variations relatives entre saisons, le modèle d'ELLIOTT et al. (1995) surestimant la croissance pondérale des truites communes.

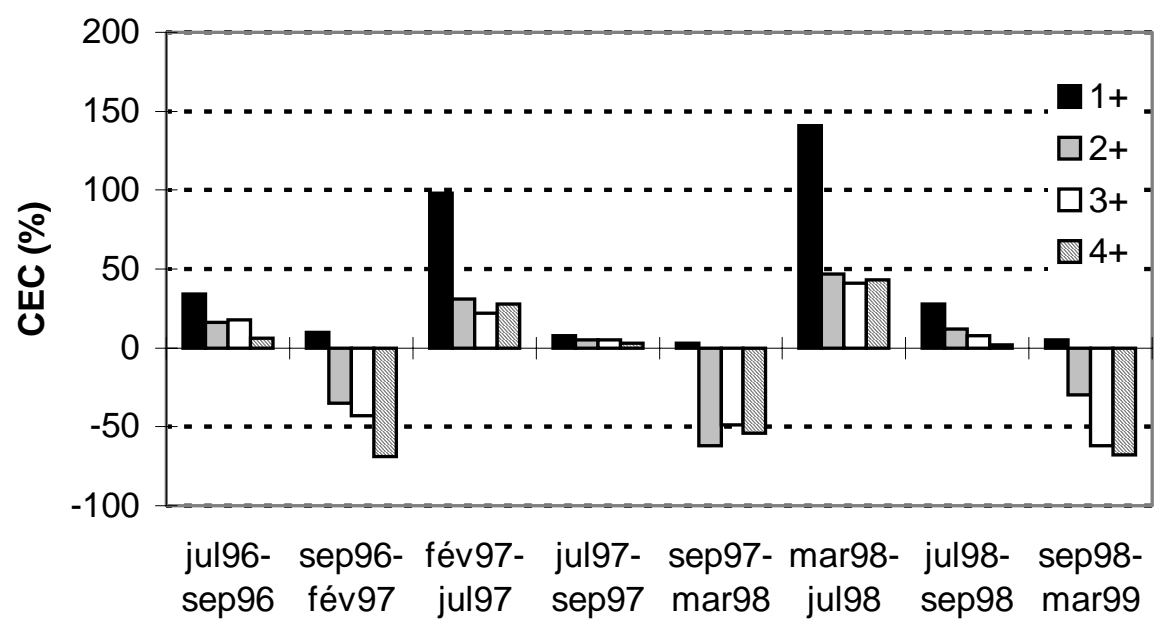

\section{Figure 6}

Evolution saisonnière des Coefficients Ecologiques de Croissance moyens par classes d'âge (CEC), évalués pour la population totale de l'ensemble des deux stations, entre juillet 1996 et mars 1999.

\section{Figure 6}

Seasonal variations of mean Ecological Growth Coefficient by age classes (EGC or $C E C$ ), evaluated for the whole total population of the two sites, between July 1996 and March 1999.

Les CEC ne sont pas significativement différents (Kruskal-Wallis, $p>0.05$ ) pour les individus d'âge $>1+$, quelle que soit la saison considérée.

A l'image des taux de croissance journaliers en poids, outre une variabilité interannuelle, il existe une évolution saisonnière très comparable d'une année à l'autre, les CEC étant significativement différents (Kruskal-Wallis, $p<0.001$ ) entre saisons, pour toutes les classes d'âge (Figure 6). Comme pour les taux de croissance journaliers en poids, les CEC sont toujours les plus élevés entre février (ou mars) et juillet, peu élevés entre juillet et septembre et faibles (pour les 1+) voire négatifs (pour les $>1+$ ) entre septembre et février (ou mars).

\section{Individus marqués : période de mars 1998 à mars 1999} marqués

Représentativité de la population et spectres de taille et d'âge des individus 
Quelles que soient la classe d'âge, la campagne et la station considérées, les tailles moyennes et les poids moyens par âge des individus marqués ne sont pas différents (Mann-Whitney, p > 0.05), ni de ceux de la population d'individus non marqués de 1998, ni de ceux de la population totale analysée en 1996 et en 1997.

Les spectres de tailles des poissons marqués aux deux stations en mars 1998 s'échelonnait entre 100 et $290 \mathrm{~mm}$, pour des âges compris entre 1+ et $6+$ ans. L'évolution du spectre d'âges des individus marqués puis recapturés, par station et par campagne et utilisés dans l'analyse qui suit, est donnée dans la Figure 7.

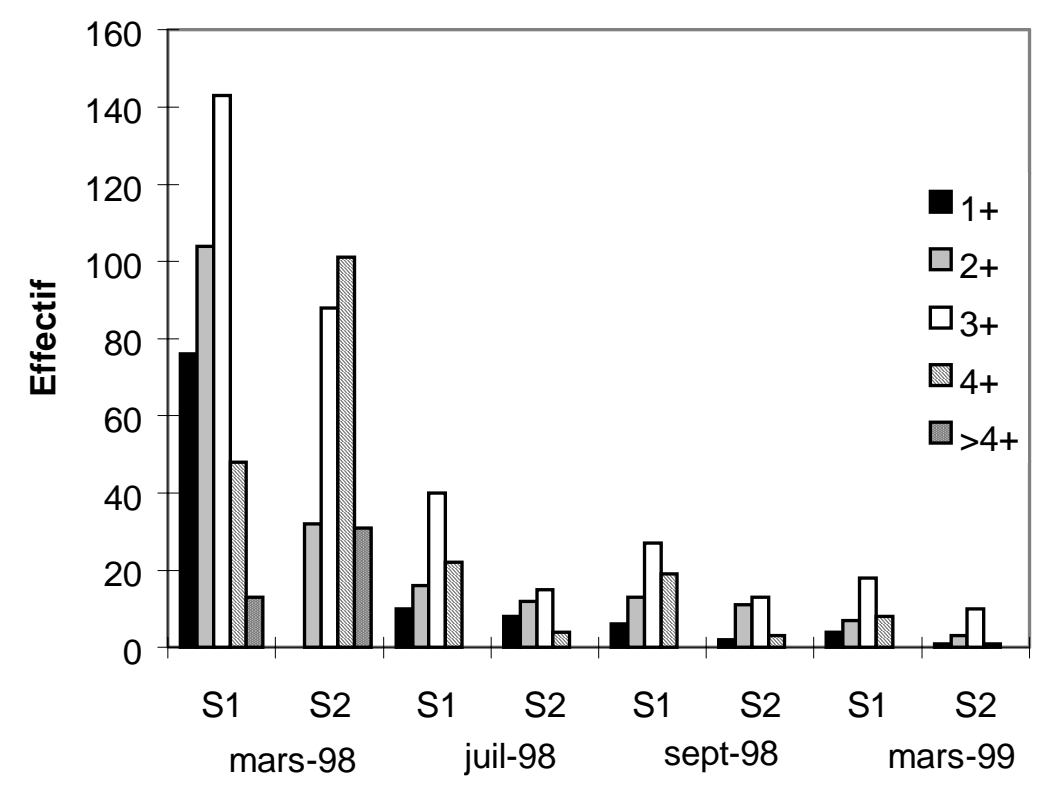

\section{Figure 7}

Evolution saisonnière des effectifs par classes d'âge d'individus marqués puis recapturés aux deux stations, entre mars 1998 et mars 1999.

\section{Figure 7}

Seasonal variation of the number of tagged and recaptured trout by age classes at the two sites, between March 1998 and March 1999.

\section{Evolution des taux de recapture des poissons marqués}

Le taux de recapture en juillet 1998 est très faible. Ce taux diminue encore en septembre 1998 et en mars 1999, mais de façon moins prononcée (Tableau III). En ce qui concerne le taux de recapture entre campagnes, celui-ci montre une baisse plus prononcée entre septembre 1998 et mars 1999 qu'entre juillet et septembre 1998. De nombreux individus n'ont pas été recapturés à toutes les campagnes.

En mars 1999, uniquement 52 individus représentant $8 \%$ des poissons marqués en mars 1998 ont été recapturés. Sur ces 52 individus, seuls 33 individus avaient été contrôlés à chacune des campagnes (24 individus pour la station 1 et 9 individus pour la station 2). 


\section{Tableau III}

Nombre d'individus marqués individuellement puis recapturés, taux de recapture par rapport à mars 1998, taux de recapture inter-campagne et intervalle intercampagne.

\section{Table III}

Number of individually tagged and recaptured trout, recapture rate compared to March 1998, recapture rate between experiments and number of days between experiments.

\begin{tabular}{|l|c|c|c|c|}
\hline Campagne & $\begin{array}{c}\text { Individus marqués* et } \\
\text { individus recapturés } \\
\text { (nbr d'ind.) }\end{array}$ & $\begin{array}{c}\text { Taux de recapture } \\
\text { depuis mars 1998 } \\
(\%)\end{array}$ & $\begin{array}{c}\text { Taux de recapture } \\
\text { entre campagnes } \\
(\%)\end{array}$ & $\begin{array}{c}\text { Intervalle } \\
\text { (nbr jours écoulés } \\
\text { depuis mars 1998) }\end{array}$ \\
\hline mars 1998 & $652^{*}$ & & & 129 \\
\hline juillet 1998 & 127 & 19.5 & 74 & 210 \\
\hline $\begin{array}{l}\text { septembre } \\
\text { 1998 }\end{array}$ & 94 & 14.4 & 55.3 & 365 \\
\hline mars 1999 & 52 & 8 & & \\
\hline
\end{tabular}

\section{Taux de croissance journalier en poids}

Pour une saison donnée et quelle que soit la station considérée, les taux de croissance journaliers en poids ne sont pas significativement différents (Mann-Whitney, $p>0.05)$, ni de ceux de la population d'individus non marqués de 1998, ni de ceux de la population totale analysée en 1996 et en 1997.

Les taux de croissance journaliers en poids ne sont pas différents (Mann-Whitney, $p>0.05)$ entre stations, quelle que soit la saison considérée. Par contre, pour une saison donnée, ils sont significativement différents entre classes d'âge (Kruskal-Wallis, $p<0.05$ ). Ils sont toujours plus élevés chez les individus d'âge 1+ (Figure 8).

II existe une évolution saisonnière marquée des taux de croissance journaliers en poids qui sont significativement différents (Kruskal-Wallis, $p<0.01$ ) entre saisons, pour toutes les classes d'âge. Cette évolution saisonnière est très comparable à celle observée lors du suivi de la population totale (Figure 4). De la même manière, le taux de croissance journalier en poids est le plus élevé entre mars et juillet 1998, peu élevé entre juillet et septembre 1998 et le plus faible entre septembre 1998 et mars 1999, pour toutes les classes d'âge (hormis pour les individus d'âge 4+ de la station 2). Au cours de cette dernière saison et comme pour le suivi de la population totale, toutes les classes d'âge $>1+$ perdent du poids, ainsi que les individus d'âge 4+ entre juillet et septembre 1998. 


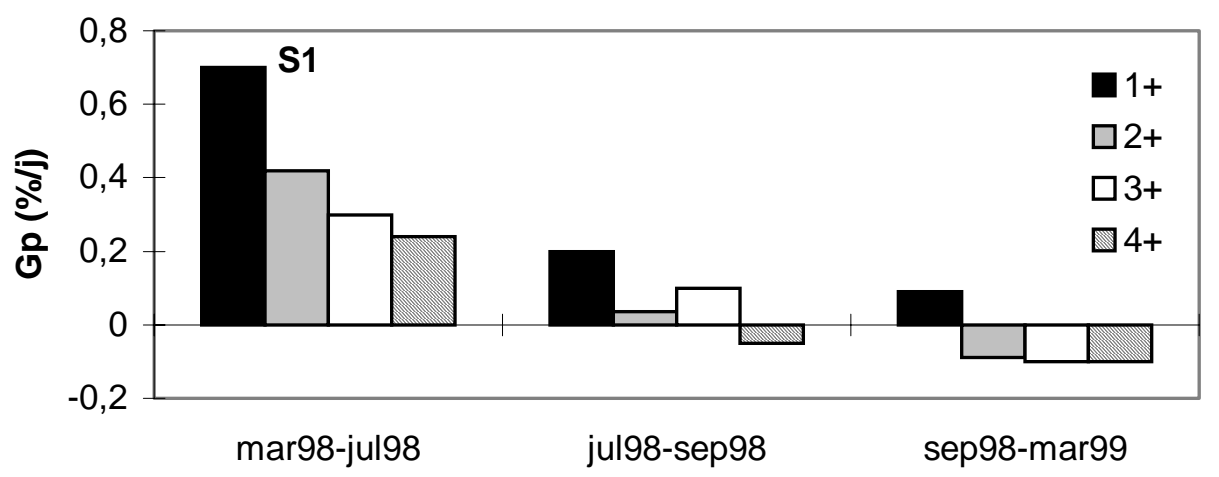

\section{Figure 8}

Evolution saisonnière des taux de croissance journaliers moyens en poids par classes d'âge (Gp), évalués pour la population d'individus marqués et aux deux stations, entre mars 1998 et mars 1999.

\section{Figure 8}

Seasonal variations of mean daily growth rates in weight by age classes (Gp), evaluated for the tagged trout population and at the two sites, between March 1998 and March 1999.

\section{Coefficients de condition}

Pour une campagne donnée et quelle que soit la station considérée, les coefficients de condition ne sont pas significativement différents (Mann-Whitney, $p>0.05$ ), ni de ceux de la population d'individus non marqués de 1998, ni de ceux de la population totale analysée en 1996 et en 1997.

Les coefficients de condition ne sont pas significativement différents, ni entre stations (Mann-Whitney, $p>0.05$ ), ni entre classes d'âge pour une campagne donnée (Kruskal-Wallis, $p>0.05)$.

Il existe une évolution saisonnière (Figure 9) très comparable à celle observée lors du suivi de la population totale (Figure 5). Similaires en juillet et en septembre, les coefficients de condition ont tendance à être plus faibles en mars, pour toutes les classes d'âge. 

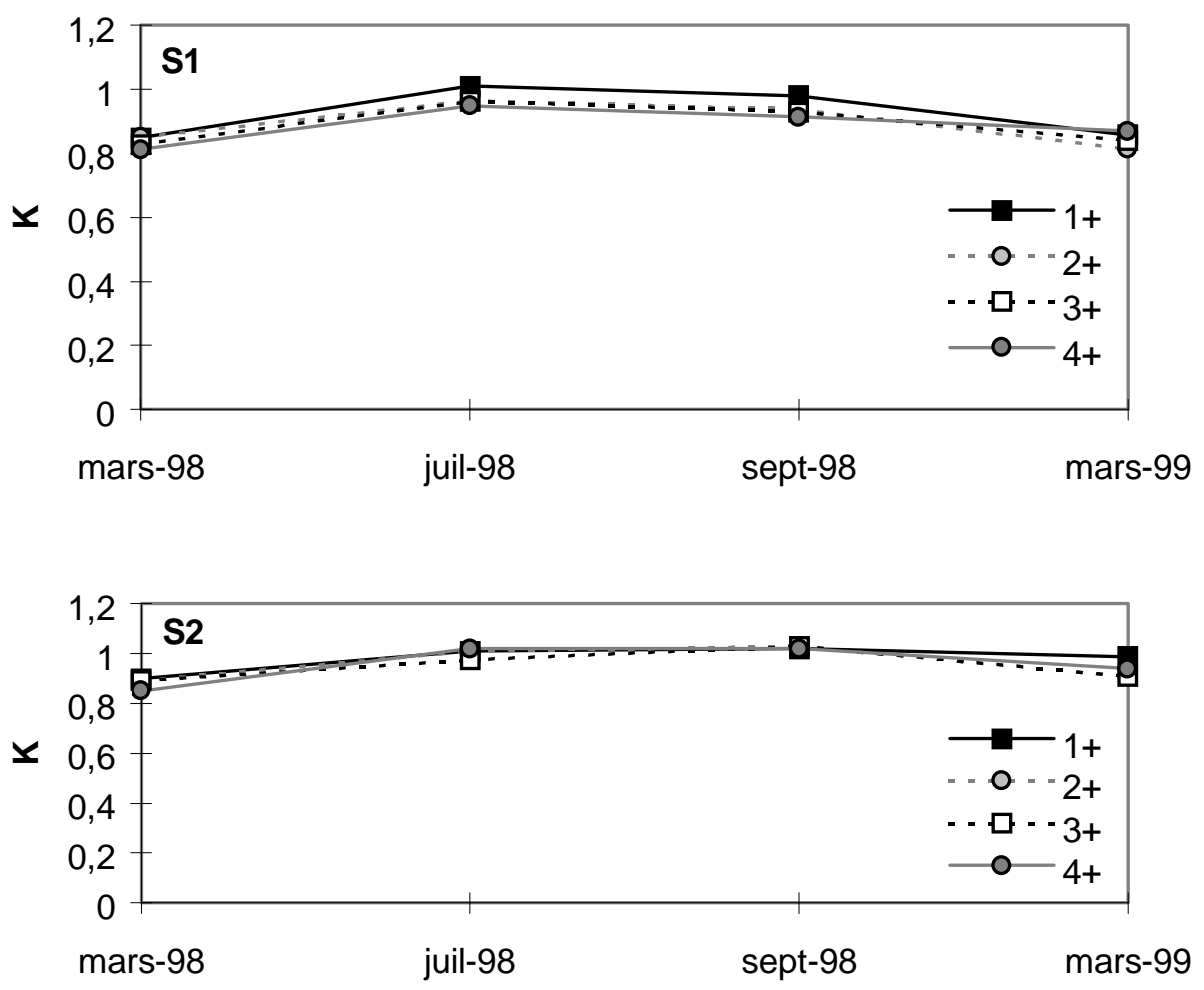

\section{Figure 9}

Evolution saisonnière des coefficients de condition moyens par classes d'âge (K), évalués pour la population d'individus marqués et aux deux stations, entre mars 1998 et mars 1999.

\section{Figure 9}

Seasonal variations of mean condition factor by age classes (K), evaluated for the tagged trout population and at the two sites, between March 1998 and March 1999.

\section{Coefficients Ecologiques de Croissance (CEC)}

A l'image de ce que nous avons fait pour le suivi de la population totale et pour les mêmes raisons, nous avons regroupé les individus marqués des deux stations pour estimer les CEC par classes d'âge d'individus marqués, pour l'ensemble du secteur de rivière étudié.

Pour une saison donnée, les $C E C$ ne sont pas significativement différents (MannWhitney, $p>0.05$ ), ni de ceux de la population d'individus non marqués de 1998, ni de ceux de la population totale analysée en 1996 et en 1997.

Comme pour le suivi de la population d'individus non marqués, les CEC sont toujours inférieurs à $100 \%$, pour toutes les saisons et pour toutes les classes d'âge, hormis pour la classe d'âge 1+ entre mars et juillet 1998 (Figure 10).

Les $C E C$ ne sont pas significativement différents (Kruskal-Wallis, $p>0.05$ ) pour les individus d'âge $>1+$, quelle que soit la saison considérée. 


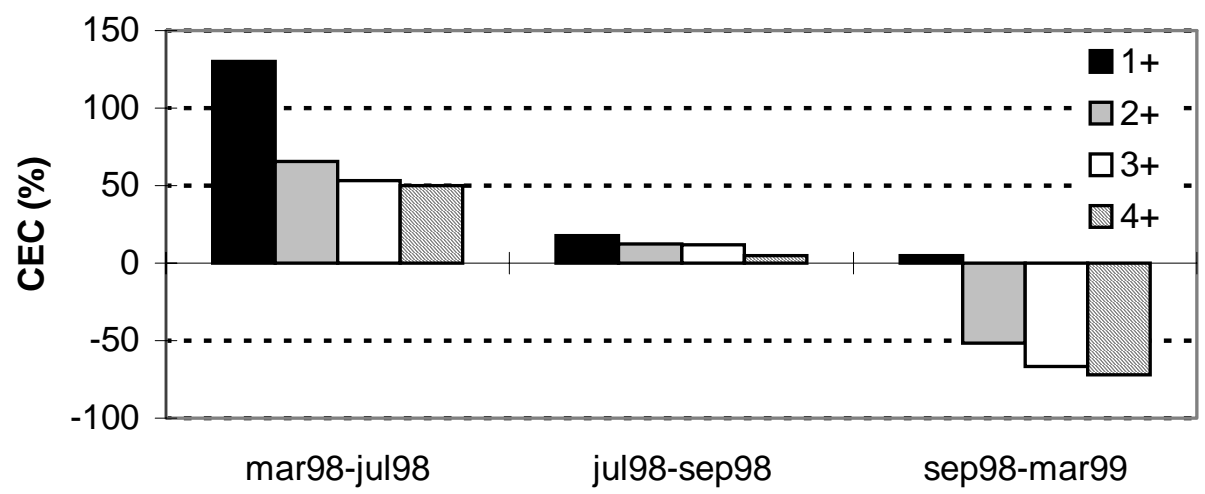

Figure 10

Evolution saisonnière des Coefficients Ecologiques de Croissance moyens par classes d'âge $(C E C)$, évalués pour l'ensemble de la population d'individus marqués des deux stations, entre mars 1998 et mars 1999.

\section{Figure 10}

Seasonal variations of mean Ecological Growth Coefficient by age classes (EGC or $C E C$ ), evaluated for the whole total population of the two sites, between March 1998 and March 1999.

A l'image des taux de croissance journaliers en poids, il existe une évolution saisonnière très comparable à celle observée lors du suivi de la population totale (Figure 6), les CEC étant significativement différents (Kruskal-Wallis, $p<0.01$ ) entre saisons, pour toutes les classes d'âge. Ils sont plus élevés entre mars et juillet 1998, peu élevés entre juillet et septembre 1998 et faibles (pour les 1+) voire négatifs (pour les $>1+$ ) entre septembre 1998 et mars 1999.

\section{DISCUSSION}

Au cours de cette étude, nous avons démontré que la croissance des truites communes d'âge $\geq 1+$ de la Neste d'Oueil présentait une forte variabilité saisonnière. Celle-ci, mise en évidence au cours du suivi de 2 ans et 9 mois de l'ensemble de la population, a été confirmée au cours d'une année de suivi par le marquage d'individus dont la population apparaît bien représentative de la population totale.

La technique du marquage individuel, très utilisée pour le suivi des populations de poissons migrateurs (BAGLINIËRE et PORCHER, 1994), élimine les biais d'une estimation de croissance saisonnière à partir de cohortes entières, dus aux mouvements et aux mortalités de poissons (RAILSBACK et ROSE, 1999). Les implants visibles que nous avons utilisés n'ont pas causé par eux-mêmes de ralentissement ou d'arrêt de croissance, les tailles moyennes et les poids moyens par âge, les taux de croissance journaliers en poids et les coefficients de condition des individus marqués n'étant pas différents de ceux des individus non marqués. C'est un avantage certain de ce type de marque déjà cité dans la littérature (BRYAN et NEY, 1994). Toutefois, ce type de marque ne peut être utilisé pour des individus de petite taille (LE LOUARN et BAGLINIĖRE, 1997). Ainsi, le suivi par marquage n'a été réalisé que pour des individus de taille $>100 \mathrm{~mm}$. L'utilisation de ces marques nous a permis de valider, au moins en partie, l'estimation de l'âge faite par la scalimétrie. Les premiers mois, le taux de recapture est faible (19.5\% de mars à juillet) et inférieur aux $50 \%$ obtenus par BRYAN et NEY (1994) sur un an chez Salvelinus fontinalis. Ceci est principalement dû aux pertes de marque dans les premiers jours suivant le 
marquage, d'où la nécessité d'un marquage de masse. Ensuite, ce taux reste élevé en période estivale $(74 \%)$ puis diminue en période automnale et hivernale (55.3\%), traduisant alors des mortalités et / ou des déplacements de poissons à cette période de l'année.

En régions tempérées, la croissance chez la truite commune est généralement forte au printemps et en été (MORTENSEN, 1985 ; LOBON-CERVIA et RINCON, 1998) puis réduite voire interrompue en hiver (EGGLISHAW et SHACKLEY, 1977 ; MORTENSEN et al., 1988 ; BERRIE et al., 1998). Cette période est critique, notamment pour les jeunes stades (CUNJAK et POWER, 1987), les poissons étant plus vulnérables à la prédation, aux maladies ou au parasitisme (BERG et BREMSET, 1998). En revanche, peu d'auteurs ont rapporté un fort ralentissement de la croissance en été, alors que les conditions du milieu, notamment du point de vue de la température, correspondent à l'optimum de développement des poissons. C'est ce que nous avons montré dans notre étude où une forte variabilité saisonnière de la croissance se traduit par une très forte croissance printanière suivie d'un net ralentissement dès la période estivale qui s'accentue durant l'automne et l'hiver.

Cette variabilité saisonnière, mise en évidence au niveau de la croissance pondérale et des coefficients de condition, résulte de l'action conjuguée de différents facteurs dont la part respective reste difficile à évaluer. MORTENSEN (1985) et PREALL et RINGLER (1989) ont également montré une évolution au cours des saisons du taux de croissance pondérale, notamment sa chute en période estivale. Une évolution comparable est relatée par BAGLINIERE et MAISSE (1990) pour le taux de croissance en taille d'individus d'âge $1+$. Pour ces auteurs, la maturation sexuelle est un facteur important de régulation du taux de croissance des individus dès l'âge 1+, sur les cours d'eau bretons. Dans les rivières des Pyrénées, on connaît peu de choses sur l'âge de première maturité des individus et sur la période de l'année où cette maturation gonadique débute. Sur la Neste d'Oueil, il semble qu'il y ait un décalage par rapport aux cours d'eau de Bretagne ou de Normandie. En effet, GOURAUD (1999) a montré sur cette rivière que l'âge de première maturité semblait être de 1+ pour les mâles et 2+ pour une partie des femelles seulement, une frange de la population de femelles $(\approx 25 \%)$ n'étant mature qu'à l'âge 3+. MAISSE et al. (1987) ont montré le rôle prépondérant de la croissance des 0+ sur la maturation précoce et l'on peut penser que la faible croissance observée sur la Neste d'Oueil soit responsable de ce décalage et influence donc indirectement la dynamique de la population.

Dans notre étude, si l'on considère uniquement les individus 1+ (qui sont immatures) et les individus 3+ et 4+ (qui sont mâtures), nous avons montré que la croissance pondérale des individus $1+$, bien que faible en automne et en hiver, restait élevée en été. Au contraire, celle des individus $3+$ et $4+$ était réduite voire négative dès l'été, suivant l'année, et négative en automne et en hiver. En outre, le calcul des CEC montre des valeurs faibles pour les individus d'âge $1+$ mais fortement négatives pour les $>1+$. Ceci s'explique certainement par le fait que le modèle thermique de croissance d'ELLIOTT et al. (1995) a été établit à partir d'individus non matures. II semble donc bien que la maturation sexuelle puisse avoir une influence sur la croissance dès la période estivale et en automne-hiver. De nombreux auteurs ont montré une influence de la maturation sexuelle sur la croissance de la truite commune (NEVEU et THIBAULT, 1977 ; CRAIG, 1982 ; MAISSE et al., 1987 ; BAGLINIERE et MAISSE, 1990 ; MAISSE et BAGLINIERE, 1990). La gamétogénèse est très consommatrice en énergie, surtout pour les femelles (BERG et al., 1998), et le développement des gonades et l'activité de reproduction perturbent l'activité alimentaire (WOOTTON, 1999).

L'effet de la maturation gonadique se retrouve dans notre étude au niveau des coefficients de condition, qui diminuent dès la fin de l'été, phénomène déjà observé (ELLIS et GOWING, 1957 ; ENSIGN et al., 1990). Les coefficients de condition suivent une 
évolution saisonnière comparable aux résultats obtenus par de nombreux auteurs (NEVEU et THIBAULT, 1977 ; NEOPHITOU, 1986 ; PREALL et RINGLER, 1989 ; ENSIGN et al., 1990).

\section{Influence de la température}

Les fonctions physiologiques liées aux flux d'énergie dépendent directement du facteur thermique (LOBON-CERVIA et RINCON, 1998). La gamme de température pour une croissance maximale de la truite commune diffère légèrement selon les études : entre $7^{\circ} \mathrm{C}$ et $19^{\circ} \mathrm{C}$ pour FROST et BROWN (1967), entre $3.9^{\circ} \mathrm{C}$ et $19.5^{\circ} \mathrm{C}$ pour ELLIOTT (1975) et entre $10^{\circ} \mathrm{C}$ et $15.5^{\circ} \mathrm{C}$ pour JOBLING (1981). Quant à l'optimum thermique de croissance pour la truite commune, il se situe, selon les études, autour de $13^{\circ} \mathrm{C}$ pour ELLIOTT (1975), $14.9^{\circ} \mathrm{C}$ pour JENSEN (1990), $13.11^{\circ} \mathrm{C}$ pour ELLIOTT et al. (1995) ou encore $13.2^{\circ} \mathrm{C}$ pour LOBON-CERVIA et RINCON (1998). Au cours des 2 ans et 9 mois de suivi sur la Neste d'Oueil, le régime thermique est resté favorable au bon développement d'une population de truite commune. Seules les températures hivernales (moyennes mensuelles entre 3 et $6^{\circ} \mathrm{C}$ ) sont pénalisantes pour la croissance et expliquent en partie l'arrêt de croissance et les pertes de poids observées en automne-hiver. Les températures printanières (moyennes mensuelles entre $8^{\circ} \mathrm{C}$ et $13^{\circ} \mathrm{C}$ ) sont plus favorables à la croissance, tandis que la période estivale se caractérise par des températures (moyennes mensuelles supérieures à $12^{\circ} \mathrm{C}$ ) proches de l'optimum thermique de croissance. Pourtant, la croissance des truites communes s'effectue en quasi totalité au printemps, indépendamment de l'optimum thermique admis. L'année 1997 est caractérisée par des températures moyennes mensuelles légèrement supérieures à celles des autres années. De même, l'écart moyen de température entre l'amont et l'aval est de $+1.1^{\circ} \mathrm{C}$ en faveur de l'aval. Pourtant, ces différences thermiques ne semblent pas se traduire par une augmentation significative de la croissance pondérale des individus.

L'utilisation du modèle thermique de croissance d'ELLIOTT et al. (1995) permettant le calcul des CEC reflète cette discordance entre croissance effective et optimum thermique, le modèle surestimant largement la croissance pondérale estivale pour toutes les classes d'âge. Sur 9 cours d'eau britanniques, EDWARDS et al. (1979) ont montré des taux de croissance observés sur une année représentant entre 60 et $90 \%$ du taux de croissance prédit selon ELLIOTT (1975). Sur 12 rivières norvégiennes, JENSEN (1990) montre des taux de croissance pondérale entre 76 et $136 \%$ des taux de croissance théoriques. Pour la période printanière, MORTENSEN et al. (1988) relèvent entre 67 et $111 \%$ des taux de croissance théoriques. Nos résultats printaniers sont assez proches, selon les classes d'âge. En revanche, les valeurs relevées en période estivale sont extrêmement faibles pour toutes les classes d'âge et notamment les $>1+$, et confirment un déficit de croissance indépendant du régime thermique.

\section{Influence de la disponibilité trophique}

MORTENSEN et al. (1988) ont relevé, entre juillet - août et novembre, des valeurs faibles de CEC (entre 23 et $70 \%$ ), mais tout de même supérieures à celles que nous obtenons. Pour expliquer cette faible conformité au modèle, ces auteurs ont émis l'hypothèse d'une limitation de la croissance par la disponibilité trophique et non par la température. De nombreux auteurs ont évoqué des limitations de croissance ou de production de salmonidés par la ressource trophique (ELLIS et GOWING, 1957 ; CADA et al., 1987 ; HUGHES et DILL, 1990 ; FILBERT et HAWKINS, 1995). RAILSBACK et ROSE (1999) ont pu montrer que le rôle de la consommation en nourriture est plus important que celui de la température dans le contrôle de la croissance estivale de truites arc-en-ciel. Quand cette ration est réduite et que la température s'élève, une grande proportion d'énergie est consommée par le métabolisme d'entretien et peu d'énergie reste disponible pour la croissance (CADA et al., 1987 ; JOBLING, 1994 ; RAILSBACK et ROSE, 1999) qui ralentit (WOOTTON, 1999). 
Bien que nécessitant des investigations plus poussées et beaucoup plus lourdes afin d'approcher le potentiel trophique de la Neste d'Oueil, les prélèvements benthiques effectués sont néanmoins révélateurs d'une présence de proies en très fortes densités en juillet mais avec des biomasses associées très faibles témoignant de proies de petite taille. Ceci n'est plus le cas en septembre où les proies certes moins nombreuses qu'en été sont de plus grande taille. La taille des proies potentiellement disponibles est importante à considérer (JENSEN, 1990), les petites proies étant généralement considérées comme peu rentables en terme d'énergie nette (après déduction de l'énergie dépensée pour leur capture (BANNON et RINGLER, 1986 ; PREALL et RINGLER, 1989).

Ainsi, la petite taille des proies potentiellement disponibles, notamment en été, semble contribuer à la faible croissance estivale observée, en dépit des conditions thermiques favorables.

\section{Influence de I'habitat physique}

Outre la nature des proies potentiellement disponibles, l'hétérogénéité de l'habitat physique contrôle l'utilisation et la répartition dans le milieu de la ressource nutritive entre les différents microhabitats occupés par les différentes classes d'âge (CADA et al., 1987). En rivière, les Salmonidés sélectionnent un microhabitat précis de compromis entre la dépense musculaire et la recherche de nourriture (FAUSH, 1984), afin d'optimiser le gain énergétique net (BACHMAN, 1984 ; FRANKIEWICZ et al., 1993 ; HEGGENES et al., 1995). En été sur la Neste d'Oueil, les débits d'étiage engendrent une diminution importante des vitesses de courant et des profondeurs. Cette diminution de la capacité d'accueil du cours d'eau peut altérer la disponibilité en habitats de nutrition énergétiquement favorables (BRAATEN et al., 1997). Cette disponibilité moindre doit influer sur les rythmes alimentaires, donc sur le niveau de satiété et la croissance des truites, cette hypothèse ayant déjà été formulée par GOURAUD (1999) sur cette même rivière.

En plus des conditions de nutrition et d'habitat, la pression exercée par les prédateurs et la compétition inter et intra-spécifique jouent un rôle important dans l'établissement de la distribution des poissons dans le cours d'eau (FRANKIEWICZ et al., 1993). Pourtant, l'influence directe de la densité sur la croissance des Salmonidés en milieu lotique montrée par certains auteurs (EGGLISHAW et SHACKLEY, 1977 ; JENKINS et al., 1999) est controversée par d'autres (revue faite par ELLIOTT, 1994). La forte croissance printanière des truites communes du cours principal assure l'essentiel de l'augmentation de la biomasse totale. De plus, en fin de printemps et en été, la densité totale a tendance à augmenter. Bien qu'abordés de façon parcellaire en raison de connaissances réduites sur la dévalaison des juvéniles des affluents vers le cours principal des rivières pyrénéennes, une arrivée d'individus des affluents (en majorité des 1+ et 2+) semble se produire dans le cours principal (GOURAUD, 1999), phénomène couramment observé dans les cours d'eau bretons et normands (MAISSE et al., 1987 ; MAISSE et BAGLINIERE, 1990, 1991 ; BAGLINIERE et MAISSE, 1990). La compétition intraspécifique devient alors maximale, les taux d'occupation de l'habitat physique sont les plus forts pour cette rivière et la capacité d'accueil du milieu physique pour la truite commune est saturée (GOURAUD, 1999). On peut donc s'attendre à une régulation de la population par des arrêts de croissance, des émigrations et / ou des mortalités de poissons (ELLIOTT, 1994), les nouveaux individus dévalants des affluents étant certainement les premiers concernés par ces phénomènes de régulation, de telle sorte que la population semble s'ajuster en permanence à la capacité d'accueil du milieu (GOURAUD, 1999). Cet auteur suggère donc l'existence d'une capacité d'accueil pour un tronçon donné autour de laquelle s'ajusterait la population. Ainsi, la diminution de la "qualité et de la quantité" d'habitat physique en été constitue un facteur limitant pour cette population. 
La croissance joue donc un rôle important dans la dynamique de la population de truite commune de la Neste d'Oueil 1) par sa forte variabilité saisonnière, 2) par son influence sur l'âge de première maturité d'une frange de la population de femelles qui semble retardé et 3) car les besoins de croissance au cours du développement ontogénique des individus des affluents semblent influencer leur émigration vers le cours principal de la rivière.

\section{REMERCIEMENTS}

Les auteurs tiennent particulièrement à remercier Véronique GOURAUD et toute la Division Recherche \& Développement d'EDF, José CAZASSUS (Garde Fédéral du secteur d'étude) et l'A.A.P.P.M.A de Luchon pour leur contribution à cette étude, ainsi que deux correcteurs anonymes pour leurs commentaires constructifs et leurs suggestions.

\section{BIBLIOGRAPHIE}

ANGELIER M.L., 1976. Le peuplement piscicole du ruisseau de la Mousquères (HautesPyrénées). Ann. Limnol., 12, 299-321.

BACHMAN R.A., 1984. Foraging behaviour of free-ranging wild and hatchery brown trout in a stream. Trans. Am. Fish. Soc., 113, 1-32.

BAGLINIERE J.L., MAISSE G., 1990. La croissance de la truite commune (Salmo trutta L.) sur le bassin du Scorff. Bull. Fr. Pêche Piscic., 318, 89-101.

BAGLINIERE J.L., PORCHER J.P., 1994. Caractéristiques des stocks de reproducteurs et comportement lors de la migration génésique. In : GUEGEN J.C., PROUZET P. (Eds.), Le saumon atlantique ; biologie et gestion de la ressource, 101-122, IFREMER, Brest.

BANNON E., RINGLER N.H., 1986. Optimal prey size for stream resident brown trout (Salmo trutta): tests of predictive models. Can. J. Zool., 64, 704-713.

BERG O.K., BREMSET G., 1998. Seasonal changes in the body composition of young riverine Atlantic salmon and brown trout. J. Fish Biol., 52, 1272-1288.

BERG O.K., THRONAES E., BREMSET G., 1998. Energetics and survival of virgin and repeat spawning brown trout (Salmo trutta). Can. J. Fish. Aquat. Sci., 55, 47-53.

BERRIE A.D., MCLEISH P.R., MACKEY A.P., 1998. Population structure, growth and biomass of brown trout, Salmo trutta, at three sites in the River Kennet, England. Pol. Arch. Hydrobiol., 45, 173-183.

BRAATEN J.P., DEY P.D., ANNEAR T.C., 1997. Development and evaluation of bioenergetic-based habitat suitability criteria for trout. Regul. Rivers: Res. Manag. 13, 345-356.

BRYAN R.D., NEY J.J., 1994. Visible implant tag retention by and effects on condition of a stream population of Brook trout. N. Am. J. Fish. Manag., 14, 216-219.

CADA G.F., LOAR J.M. SALE M.J., 1987. Evidence of food limitation of Rainbow and Brown trout in southern Appalachian soft-water streams. Trans. Am. Fish. Soc., 116, 692-702.

CHAUVET E., 1983. Influence d'une réduction de débit sur un torrent de montagne : l'Aston (Ariège). Ann. Limnol., 19, 45-49.

CRAIG J.F., 1982. A note on growth and mortality of trout, Salmo trutta L., in afferent streams of Windermere. J. Fish Biol., 20, 423-429.

CUNJAK R.A., POWER G., 1987. Winter habitat utilisation by stream resident brook trout (S. fontinalis) and brown trout (S. trutta). Can. J. Fish. Aquat. Sci., 43, 1970-1981.

DELACOSTE M., BARAN P., LEK S., LASCAUX J.M., 1995. Classification et clé de détermination des faciès d'écoulement en rivière de montagne. Bull. Fr. Pêche Piscic., 337/338/339, 149-156. 
DE LURY D.B., 1951. On the planning of experiments for the estimation of fish populations. J. Fish. Res. Bd. Can., 18, 281-307.

EDWARDS R.W., DENSEM J.W., RUSSELL P.A., 1979. An assessment of the importance of temperature as a factor controlling the growth of brown trout in streams. J. Anim. Ecol., 48, 501-507.

EGGLISHAW H.J., SHACKLEY P.E., 1977. Growth, survival and production of juvenile salmon and trout in a Scottish stream. J. Fish Biol., 11, 647-672.

ELLIOTT J.M., 1975. The growth rate of brown trout (Salmo trutta L.) fed on maximum rations. J. Anim. Ecol., 44, 805-821.

ELLIOTT J.M., 1994. Quantitative Ecology and the Brown Trout. Oxford Univ. Press, Oxford, $286 \mathrm{p}$.

ELLIOTT J.M., HURLEY M.A., FRYER R.J., 1995. A new, improved growth model for brown trout, Salmo trutta. Funct. Ecol., 9, 290-298.

ELLIS R.J., GOWING H., 1957. Relationship between food supply and condition of wild Brown trout, Salmo trutta L., in a Michigan stream. Limnol.Oceanogr., 11, 299-308.

ENSIGN W.E., STRANGE R.J., MOORE S.E., 1990. Summer food limitation reduces Brook and Rainbow trout biomass in a southern Appalachian stream. Trans. Am. Fish. Soc., 119, 894-901.

FAUSCH K.D., 1984. Profitable stream positions for salmonids: relating specific growth rate to net energy gain. Can. J. Zool., 62, 441-451.

FILBERT R.B., HAWKINS C.P., 1995. Variation in condition of rainbow trout in relation to food, temperature, and individual length in the Green River, Utah. Trans. Am. Fish. Soc., 124, 824-835.

FRANKIEWICZ P., ZALEWSKI M. THORPE J.E., 1993. Feeding pattern of brown trout (Salmo trutta L.) from the river Earn (Scotland), in relation to invertebrate drift. Pol. Arch. Hydrobiol., 40, 15-29.

FROST W.E., BROWN M.E., 1967. The trout. Collins, St James Place, London, 286 p.

GOURAUD V., 1999. Etude de la dynamique de populations de truite commune (Salmo trutta L.) à l'aide d'un modèle déterministe. Thèse doc., Ecole Nationale du Génie Rural, des Eaux et Forêts de Paris, $166 \mathrm{p}$.

GOURAUD V., BARAN P., LIM P., SABATON C., 1999. Dynamics of a population of brown trout (Salmo trutta) and fluctuations in physical habitat conditions experiments on a stream in the Pyrenees ; first results. In : COWX I.G. (Eds.), River Fisheries, Fishing News Books, Blackwell Science, Oxford.

HEGGENES J., BAGLINIERE J.L., CUNJAK R., 1995. Note de synthèse sur la sélection de niche spatiale et la compétition chez le jeune saumon atlantique (Salmo salar) et la truite commune (Salmo trutta) en milieu lotique. Bull. Fr. Pêche Piscic., 337/338/339, 231-239.

HUGHES N.F., DILL L.M., 1990. Position choice by drift-feeding salmonids : model and test for arctic grayling (Thymallus arcticus) in subarctic mountain streams, interior Alaska. Can. J. Fish. Aquat. Sci., 47, 2039-2048.

JENKINS T.M., DIEHL S., KRATZ K.W., COOPER S.D., 1999. Effects of population density on individual growth of brown trout in streams. Ecology, 80, 941-956.

JENSEN A.J., 1990. Growth of young migratory brown trout (Salmo trutta) correlated with water temperature in Norwegian rivers. J. Anim. Ecol., 59, 603-614.

JOBLING M., 1981. Temperature tolerance and final preferendum - rapid methods for the assessment of optimum growth temperatures. J. Fish. Biol., 19, 439-455.

JOBLING M., 1994. Fish bioenergetics. CHAPMAN and HALL (Eds.), London, $293 \mathrm{p.}$

LAGARRIGUE T., BARAN P., LASCAUX J.M., DELACOSTE M., ABAD N., LIM P., 2001. Croissance de la truite commune (Salmo trutta L.) dans les rivières des Pyrénées françaises : relations avec les caractéristiques mésologiques et influence des aménagements hydroélectriques. Bull. Fr. Pêche Piscic., (sous presse).

LE LOUARN H., BAGLINIERE J.L., 1997. Apports et limites du marquage individuel dans la connaissance de l'écobiologie du chevaine (Leuciscus cephalus). Bull. Fr. Pêche Piscic., 346, 557-571. 
LOBON-CERVIA J., RINCON P.A., 1998. Field assessment of the influence of temperature on growth rate in brown trout population. Trans. Am. Fish. Soc., 127, 718-728.

MAISSE G., BAGLINIERE J.L., LEBAIL P.Y., 1987. Dynamique de population de truite commune (Salmo trutta L.) d'un ruisseau breton (France) : les géniteurs sédentaires. Hydrobiologia, 148, 123-130.

MAISSE G., BAGLINIERE J.L., 1990. The biology of brown trout, Salmo trutta L., in the river Scorff, Britanny : a synthesis of studies from 1973 to 1984. Aquacul. Fish. Manage., 21, 95-106.

MAISSE G., BAGLINIERE J.L., 1991. Biologie de la truite commune (Salmo trutta L.) dans les rivières françaises. In : BAGLINIERE J.L., MAISSE G. (Eds.), La truite, biologie et écologie, 25-45, Edition INRA, Paris.

MORTENSEN E., 1985. Population and energy dynamics of trout Salmo trutta in a small Danish stream. J. Anim. Ecol., 54, 869-882.

MORTENSEN E., GEERTZ-HANSEN P. MARCUS E. 1988. The significance of temperature and food as factors affecting the growth of Brown trout (Salmo trutta L.) in four Danish streams. Pol. Arch. Hydrobiol., 35, 533-544.

NEOPHITOU C., 1986. Growth and population structure of brown trout, Salmo trutta fario L., in homeothermous stream conditions from a management point of view. Aquac. Fish. Manage., 17, 299-311.

NEVEU A., THIBAULT M., 1977. Comportement alimentaire d'une population sauvage de truites fario (Salmo trutta L.) dans un ruisseau des Pyrénées atlantiques, le Lissuraga. Ann. Hydrobiol., 8, 111-128.

OMBREDANE D., BAGLINIERE J.L., 1992. Les écailles et leur utilisation en écologie aquatique. In : BAGLINIERE J.L., CASTANET J., CONAND F., MEUNIER F.J. (Eds.), Tissus durs et âge individuel des vertébrés, 151-192, ORSTOM-INRA, Paris.

PREALL R.J., RINGLER N.H., 1989. Comparison of actual and potential growth rates of brown trout (Salmo trutta) in natural streams based on bioenergetic models. Can. J. Fish. Aquat. Sci., 46, 1067-1076.

RAILSBACK S.F., ROSE K.A., 1999. Bioenergetics modeling of stream trout growth : temperature and food consumption effects. Trans. Am. Fish. Soc., 128, 241-256.

SEBER G.A.F., LECREN E.D., 1967. Estimating population parameters from catches large relative to the population. J. Anim. Ecol., 36, 631-643.

SOKAL R.R., ROHLF F.J., 1997. Biometry-The principles and practice of statistics in biological research-Third edition. FREEMAN W. H., and Company (Eds), New York, $887 \mathrm{p}$.

WOOTTON R.J., 1999. Ecology of Teleost Fishes. $2^{\text {nd }}$ ed. WOOTTON R.J. (Eds.), Kluwer Academic Publishers, $364 \mathrm{p}$. 